\title{
Religion et nationalisme dans les manuels de lecture courante des Frères du Sacré-Coeur du Canada français (1897-1910)
}

\section{Jacques Paradis}

Volume 47, 1980

URI : https://id.erudit.org/iderudit/1007109ar

DOI : https://doi.org/10.7202/1007109ar

Aller au sommaire du numéro

Éditeur(s)

Les Éditions Historia Ecclesiæ Catholicæ Canadensis Inc.

ISSN

0318-6172 (imprimé)

1927-7067 (numérique)

Découvrir la revue

Citer cet article

Paradis, J. (1980). Religion et nationalisme dans les manuels de lecture courante des Frères du Sacré-Coeur du Canada français (1897-1910). Sessions d'étude - Société canadienne d'histoire de l'Église catholique, 47, 75-94.

https://doi.org/10.7202/1007109ar

Tous droits réservés @ Les Éditions Historia Ecclesiæ Catholicæ Canadensis Inc., 1980
Ce document est protégé par la loi sur le droit d'auteur. L'utilisation des services d'Érudit (y compris la reproduction) est assujettie à sa politique d'utilisation que vous pouvez consulter en ligne. 
s.C.H.E.C., Sessions d'études, 47 (1980, pp. 75-93)

\section{Religion et nationalisme dans les manuels de lecture courante des Frères du Sacré-Cceur du Canada français (1897-1910) *}

L'étude des manuels scolaires n'a pas tellement retenu l'attention des chercheurs québécois. Jusqu'à maintenant ce sont surtout les manuels d'histoire qui ont été l'objet d'analyse ${ }^{1}$. Pourtant le contenu de ces ouvrages s'avère un excellent reflet des idéologies d'une société.

Le livre scolaire touche les élèves de toutes les classes sociales indépendamment du revenu de leurs parents. Sa réalisation implique plusieurs groupes sociaux. D'une part, les auteurs mettent à l'œuvre leur méthode d'enseignement en tenant compte des directives pour l'approbation des manuels. D'autre part, les responsables de l'éducation exercent un contrôle principalement sur le contenu des volumes en accord avec l'idéologie dominante du milieu et les exigences de la pédagogie. Les débats engendrés autour des manuels scolaires publiés au Québec confirment leur impact social.

Dans ce travail, j'étudierai la série des manuels de lecture française rédigée par les Frères du Sacré-Cœur et qui couvre la période de 1897 à 1910. Dans une première partie, je me limiterai à décrire le processus d'approbation des manuels scolaires pour mieux faire ressortir le contexte social de cette époque et ainsi faciliter leur interprétation. En second lieu, j’analyserai le contenu des manuels (illustrations, auteurs et textes) sous l'angle de la religion : doctrine et vie morale. Enfin, j'examinerai plus en détail le nationalisme prôné, car c'est par ce phénomène que s'effectueront davantage les rapprochements entre la foi et la patrie au Canada français vers 1900 .

* Je remercie $\mathbf{M}$. Guy Laperrière pour sa collaboration à la révision de ce texte, dont je conserve toute la responsabilité.

1 Geneviève LalouX-JaIN, Les manuels d'histoire du Canada au Québec et en Ontario de 1867 à 1914, Québec, Presses de l'Université Laval, 1974, 250 p. Coll. : Histoire et sociologie de la culture, no 5 ; Sylvie Vincent et Bernard ARCAND, L'image de l'Amérindien dans les manuels scolaires du Québec, Montréal, Hurtubise HMH, 1979, 334 p. Coll.: Cultures amérindiennes, Cahiers du Québec, no 51. 


\section{I — L'APPROBATION DES MANUELS}

Il importe de décrire le mécanisme d'approbation des manuels scolaires afin de mettre en relief les implications religieuses et sociales d'une telle action. Dans la Province de Québec, gouvernée par les libéraux de 1897 à 1920, le principal organisme responsable de l'éducation était le Département de l'Instruction publique. Il avait à sa tête un Surintendant qui présidait le Conseil de l'Instruction publique constitué des Comités catholique et protestant. Chaque comité était entièrement responsable de prendre les décisions qui concernaient sa confession religieuse. Si, depuis sa fondation, le Conseil s'est réuni à quelques reprises, à partir de 1908, chaque comité travaillera séparément jusqu'en 19602 .

Institué en 1869 et réorganisé en 1875, le Comité catholique supervisait l'enseignement au Québec. Il était composé, d'une part, de tous les évêques ou de leurs représentants qui avaient leur diocèse en tout en partie dans la Province de Québec et, d'autre part. d'un nombre égal de laïcs nommés par le lieutenant-gouverneur en Conseil. Une des fonctions du Comité catholique consistait à approuver les manuels scolaires destinés aux écoles catholiques et,à cet effet, il édictait des normes pour guider les éditeurs. Le Comité tenait à ce que les livres mis à la disposition des élèves soient conformes aux principes de l'éducation chrétienne. Le Comité catholique avait formé un sous-comité pour étudier les demandes d'approbation de 3 manuels scolaires. Présidé le plus souvent par un évêque et composé de membres du clergé et de laïcs, ce sous-comité avait pour but d'examiner les livres au plan pédagogique et moral. Il pouvait aussi recourir à la consultation d'experts. Ayant rédigé son rapport, il le soumettait au Comité catholique qui prenait alors une décision finale.

Quelles étaient les grandes orientations de cet organisme suprême en matière d'éducation? Étant donné sa composition, l'influence cléricale s'exerçait évidemment. Il convient donc de rappeler la pensée des évêques à propos de l'éducation catholique. Vers la fin du XIX siècle, ceux-ci prennent des positions claires sur différents problèmes d'éducation: projet d'établissement d'un Ministère de l'Instruction publique, projet d'uniformisation et de gratuité des manuels scolaires, querelle de l'instruction obligatoire. En général,

2 Ls-Philippe Audet, Histoire de l'enseignement al Québec, 1608 1971, Montréal, Holt, Rinehart et Winston Ltée, 1971. Tome 2: 1840 1971, p. 341. Ce volume constitue la principale source utilisée dans ce travail en ce qui concerne l'organisation scolaire au Québec. 
à propos de ces trois sujets controversés, ils adoptent une attitude défensive toujours prête à se changer en une action vigoureuse ${ }^{3}$.

La pensée des auteurs des manuels analysés s'identifie à celle de l'Église dont fait partie leur Institut. Celui-ci a été fondé à Lyon en 1821 dans le but spécifique, précisent les Règles de 1867, d' "élever chrétiennement la jeunesse, spécialement les enfants des campagnes et des petites villes ${ }^{4}$. Dans un manuel édité en 1913 et utilisé pour la formation religieuse des frères, on trouve une riche synthèse de la pensée de cet Institut sur l'éducation chrétienne :

L'éducation, c'est la direction donnée à une âme pour l'élever vers Dieu et la préparer à la vocation que le ciel lui destine. L'éducation développe les germes de bien déposés par Dieu dans le cœur de l'enfant, atténue les suites du péché, supprime ce qui est excessif et dirige vers l'acquisition du bien suprême toutes les forces placées en nous par une Providence infiniment généreuse.

Quelle œuvre sublime! Mais, en conséquence, combien noble, combien sublime aussi, combien parfait doit être l'heureux instrument de ce travail incomparable 5 .

C'est dans cet état d'esprit que les FF. Théodule et Henri, membres de cet Institut religieux implanté au Canada en 1872, rédigèrent leurs manuels. Théodule Tressol (Fr. Théodule), Français, maitre des novices (1878-1900) et provincial (1900-1912) de la province communautaire du Canada, se consacra à la publication de nombreux manuels. "Déjà il avait dirigé la composition de manuels de lecture, de grammaire, de rédaction et d'anglais ${ }^{6}{ }^{6}$. Dans son histoire

3 Voir Ls-Philippe AUdeT, op. cit., pp. 247-256. Aussi Henri BERNARD, La Ligue de l'Enseignement, histoire d'une conspiration maçonnique à Montréal, Montréal, s. éd., 1904 ; T.D. Bouchard, L'instruction obligatoire, Saint-Hyacinthe, imp. Yamaska, 1912 ; Lionel Groulx, L'enseignement français au Canada, Montréal, Granger, 1934. Tome I : Dans le Québec, pp. 273-315 ; Hermas LALANDE, L'instruction obligatoire; principes et conséquences, Montréal, imp. du Messager, 1919; Victrice LeSSARD, L'instruction obligatoire dans la province de Québec de 1875 à 1945. Thèse non publiée de doctorat en éducation (Ph.D.) à l'Université d'Ottawa, 1962 ; Charles-Joseph Magnan, $A$ propos d'éducation obligatoire, Québec, s. éd., 1919.

4 Règles générales et communes de l'Institut des Frères de l'Instruction chrétienne des Sacrés-Cours, Le Puy (France), Typo. et Litho. M.-P. Marchessou, 1867, p. 5.

5 (FrÈre ARThÈME), Manuel de perfection chrétienne et religieuse, Paris, Desclée, De Brouwer et Cie, 1913, p. 518.

6 Frère Stanislas, Les Frères du Sacré-Caur, historique de l'Institut, 1821-1956, Rome, Les Frères du Sacré-Cœur, 1956, p. 208. 
des Frères du Sacré-Cœur au Canada, il déclare avoir composé, avec le Frère Henri, un syllabaire et des tableaux de lecture ainsi que trois volumes de lecture pour les cours élémentaire, moyen et supérieur ${ }^{7}$. Quant à Phidime Dion (Fr. Henri), né à Montmagny, il fit partie du groupe fondateur du Noviciat, en 1878, à Arthabaskaville. C'était «un homme cultivé qui a beaucoup contribué à promouvoir les études chez nos frères $\gg 8$.

À cette époque, plusieurs communautés religieuses enseignantes utilisaient leurs propres manuels dans les écoles qu'elles dirigeaient. Des clauses dans leur contrat avec les commissions scolaires stipulaient qu'elles pouvaient recourir à leurs méthodes et à leurs manuels ${ }^{9}$. De plus, il existait une certaine concurrence entre les instituts religieux d'enseignants à ce propos. Il aurait été inopportun d'employer un ouvrage publié par une autre communauté ${ }^{10}$. Ainsi, d'une

7 (Frère Théodule), Les Frères du Sacré-Caur au Canada, 1872. 1936, s.1., s. éd., (1936), p. 138.

8 (Frère Stanislas), Nécrologe de l'Institut, 1821-1961, Rome, Les Frères du Sacré-Cœur, (1962), livre non paginé, voir le 29 novembre.

9 «Les Frères donneront un bon cours commercial, enseignant avec leurs livres et selon les méthodes généralement en vigueur dans leurs établissements. Ils pourront vivre au sein de leur communauté conformément à leurs règles et à la direction des supérieurs de leur congrégation». Article 14 du contrat passé entre la commission scolaire d'Arthabaska et les Frères des Écoles chrétiennes, 1905, et reproduit dans le manuscrit de Mgr L.-A. CôTÉ, Une page de l'histoire d'Arthabaska, conservé aux archives du Séminaire de Nicolet et cité par Jules MARTEL, Histoire du Collège de Victoriaville, Victoriaville, s. éd., 1965, Tome I : Arthabaska (1872-1905), Annexe XXII, p. 53. C'est un contrat type.

10 Le $1^{\mathrm{er}}$ septembre 1928, les commissaires d'écoles de St-Hyacinthe rappelaient aux Frères du Sacré-Cœur de l'Académie Girouard qu'ils devraient utiliser le manuel anglais recommandé en 1918 :

«Le président fait rapport que le Frère Directeur de l'Académie Girouard a changé le manuel «La classe en anglais » par les Frères de l'Institution Chrétienne (sic) pour celui des Frères du Sacré-Cœur.

Attendu que cette commission a adopté l'uniformité des livres pour ses écoles pour des raisons que tout le monde connaît.

Attendu que les Commissaires ne voient pas l'utilité ni l'opportunité de changer le manuel «La classe en anglais » fait par les Frères de l'Institution Chrétienne ( sic) pour celui fait par les Frères du Sacré-Cœur ;

Il est proposé par le Commissaire Solis et unanimement résolu que le secrétaire-trésorier soit autorisé d'aviser le Frère Directeur de l'Académie Girouard d'avoir à continuer l'enseignement de l'anglais par le manuel approuvé par cette commission et échanger pour ce dernier le manuel nouvellement distribué ».

(Signés) Henri Morin, sec. Samuel Casavant, prés. 
façon générale, pour étudier la répartition des manuels scolaires publiés par une congrégation religieuse, il suffit de suivre son implantation dans le milieu québécois. En ce qui concerne les Frères du Sacré-Cœur, en 1898, ils comptent une centaine de membres professant à 2,970 élèves dans quatorze écoles situées en majorité dans les Cantons de l'Est, les Bois-Francs et Saint-Hyacinthe ${ }^{11}$. Pour évaluer l'importance des communautés religieuses enseignantes dans la province de Québec, il faut se rappeler qu'en 1899-1900, elles constituent $44 \%(3,958)$ du personnel enseignant ; les laïcs forment $56 \%$, soit $5,109^{12}$.

L'approbation des manuels de lecture courante des Frères du Sacré-Cocur a suivi les différentes étapes prescrites par le Comité catholique. En 1897, le Frère Théodule envoyait plusieurs volumes au Département de l'Instruction publique en vue de leur adoption : «Une série de Tableaux de lecture comprenant 14 tableaux; un Syllabaire; un livre intitulé : Livre de lecture courante (cours élémentaire); un livre intitulé : Livre de lecture courante (cours moyen) » ${ }^{13}$. Le prix des manuels était mentionné : tableaux, $\$ 1.00$ la série et $\$ 9.00$ la douzaine ; syllabaire, $15 \phi$ et $\$ 1.20$ la douzaine; cours moyen, $25 \phi$ et $\$ 2.50$ la douzaine.

Quelque temps après, le Frère Népotien, d'Arthabaskaville, écrivait à $\mathrm{M}^{\mathrm{gr}}$ Ls-Zéphirin Moreau, évêque de Saint-Hyacinthe, pour l'avertir que les Frères du Sacré-Cœur avaient soumis des livres à l'approbation du Conseil de l'Instruction publique, et les recommander à son attention ${ }^{14}$. À Québec, les diverses étapes de l'approbation se poursuivaient. Le sous-comité chargé de l'examen des livres présenta son rapport au Comité catholique, recommandant qu'ils «soient examinés par des personnes choisies par le Surintendant, lesquelles devront faire rapport sur les mérites de ces livres et tableaux de lecture à ce sous-comité »15. Le Comité catholique adopta cette

11 Le Canada ecclésiastique, almanach annuaire du clergé canadien, Montréal, Cadieux et Derome, 1899, vol. 13, pp. 150-151. Ces chiffres du Canada ecclésiastique sont sujets à caution.

12 Ls-Philippe AudET, op. cit., p. 319.

13 Lettre du Frère Théodule à Pierre Boucher de La Bruère, Arthabaskaville, 17 avril 1897, p. 1. Archives nationales du Québec (désormais ANQ), E-2-487.

14 Lettre du Frère Népotien à Mrr Ls-Zéphirin Moreau, Arthabaskaville, $1^{\mathrm{er}}$ mai 1897 , p. 1. Archives de l'évêché de Saint-Hyacinthe. Dossier : Frères du Sacré-Cœur, VIII-B-5.

15 Rapport du Surintendant de l'Instruction publique de la Province de Québec pour l'année 1896-1897. Québec, Charles Pageau, 1897. Appendice 
recommandation à sa séance du 19 mai 1897. Quelques jours plus tard, le Surintendant communiqua la résolution du sous-comité au Frère Théodule lui demandant que les Frères se rendent « responsables du coût de cet examen », ce que celui-ci accepta le 25 août ${ }^{16}$.

À la suite du rapport de l'expert consulté, le sous-comité fit le compte rendu suivant au Comité catholique, le 11 mai 1898 :

1. Syllabaire, "Livre de lecture courante, cours élémentaire ", "Livre de lecture courante, cours moyen" et 14 tableaux, par les Frères du Sacré-Cœur.

Le sous-comité est d'avis que ces livres sont très bien faits et sont très recommandables. Quant aux tableaux qui accompagnent ces livres, le sous-comité ne croit pas devoir les recommander 17 .

Dans le procès-verbal du Comité catholique, on ne trouve aucune résolution acceptant ou refusant ce rapport. On apprend son approbation dans une lettre du Surintendant au Frère Théodule par laquelle il lui communiquait les recommandations du comité ${ }^{18}$. Les procédures ont été similaires pour l'approbation du livre de lecture courante, cours supérieur, en $1910^{19}$.

Cette série de manuels de lecture française s'adressait aux élèves du cours primaire, c'est-à-dire à des garçons âgés de 6 à 14 ans et même davantage ${ }^{20}$. Comme les programmes étaient chargés,

$\mathrm{n}^{\mathrm{o}}$ VII. Procès-verbaux du Conseil de l'Instruction publique et des comités catholique et protestant, p. 319.

16 Correspondance conservée aux ANQ E-2-487.

17 Rapport du Surintendant de l'Instruction publique de la Province de Québec pour l'année 1897-1898. Québec, Charles Pageau, 1898. Appendice $\mathrm{n}^{\mathrm{O}} \mathrm{IX}$, Conseil de l'Instruction publique : procès-verbaux des comités catholique et protestant, 7 septembre 1897, p. 343.

18 Lettre du Surintendant de l'Instruction publique au Frère Théodule, Québec, 16 mai 1898. ANQ E-2-487. (copie).

19 Rapport du Surintendant de l'Instruction publique de la Province de Québec pour l'année 1909-1910. Québec, Louis-V. Filteau, 1910. Appendice $\mathrm{n}^{\circ}$ VIII. Procès-verbaux des comités catholique et protestant du Conseil de l'Instruction publique, 11 mai 1910, pp. 456-457.

20 Cette série était utilisée pour tout le cours primaire : Tableaux et syllabaire, $1^{\text {re }}$ année ; cours élémentaire, $2^{\mathrm{e}}$ année ; cours moyen, $3^{\mathrm{e}}$ et $4^{\mathrm{e}}$ années ; cours supérieur, 5e, 6e, $7^{\mathrm{e}}$ et $8^{\mathrm{e}}$ années. Cf. Liste des livres à employer dans les Écoles de St-Hyacinthe, à partir du 1er septembre 1918, dans Livre des délibérations des commissaires d'écoles pour la municipalité de la ville de St-Hyacinthe, vol. 2, p. 212. Archives de la Commission scolaire locale de St-Hyacinthe. 
on prenait parfois deux ou trois ans de plus pour suivre le cours primaire échelonné sur huit ans : cours élémentaire, 4 ans ; cours modèle et cours académique, 2 ans chacun ${ }^{21}$. Il en sera ainsi de la division de l'enseignement primaire public au Québec de 1888 à 1923 22. Selon C.-J. Magnan, les élèves catholiques du secteur public inscrits dans les huit années du cours se répartissaient comme suit en 1910-1911: $1^{\text {re }}$ année, $40 \% ; 2^{\mathrm{e}}$ année, $24 \% ; 3^{\mathrm{e}}$ année, $18 \% ; 4^{\mathrm{e}}$ année, $9.4 \% ; 5^{\mathrm{e}}$ année, $4 \% ; 6^{\mathrm{e}}$ année, $2 \% ; 7^{\mathrm{e}}$ année, $0.8 \% ; 8^{\mathrm{a}}$ année, $0.4 \%$. De plus, le taux de l'assiduité est intéressant à considérer. Pour l'année 1915-1916, selon T.-D. Bouchard, il se présentait ainsi pour les étudiants catholiques de l'enseignement public : $1^{\mathrm{re}}$ année, $76.3 \% ; 2^{\mathrm{e}}$ année, $86.66 \% ; 3^{\mathrm{e}}$ année, $90.10 \% ; 4^{\mathrm{e}}$ année, $90.24 \% ; 5^{\circ}$ année, $89.66 \% ; 6^{\circ}$ année, $84.6 \% ; 7^{\mathrm{e}}$ année, $73.59 \%{ }^{23}$. Ces données sur les effectifs scolaires permettront de mieux saisir l'influence du contenu idéologique des volumes étudiés.

\section{II - LA RELIGION DANS LES MANUELS}

Une analyse des volumes retenus ${ }^{24}$ permet de constater que l'enseignement religieux imprégnait une des principales matières du programme scolaire, le cours de français. Le nombre de leçons portant sur la religion confirme cette emprise : cours élémentaire, $23.7 \%$; cours moyen, $29.7 \%$; cours supérieur, $21.6 \%$. Comme on l'a vu, la conception chrétienne de l'éducation inspire la vie scolaire de l'époque. À travers l'illustration, le choix des auteurs cités et des sujets abordés, nous circonscrirons l'enseignement doctrinal et moral du catholicisme transmis aux élèves des classes primaires au tournant du siècle.

Les illustrations concernant la doctrine sont moins nombreuses que celles se référant à la vie morale. En général, les premières

21 Ls-Philippe Audet, op. cit., p. 273. Citant Gérard Filteau, Le système scolaire de la province de Québec, Montréal, Le Centre de Psychologie et de Pédagogie, 1954, pp. 79-87.

22 Ibid., p. 273.

23 Ibid., p. 280.

24 Rappelons-en les titres : (Théodule, Frère et Frère Henri), Méthode de lecture par les Frères du Sacré-Cæur, Syllabaire, Montréal, Les Frères du Sacré-Cœur, 1897, 48 p., ill.; Livre de lecture courante par les Frères du Sacré-Cour, cours élémentaire, 3* éd., Montréal, Les Frères du Sacré-Cœur, 1910, 160 p., ill ; Livre de lecture courante par les Frères du Sacré-Cour, cours moyen, (2* éd.), Montréal, Les Frères du Sacré-Cœur, 1897, 272 p., ill. ; Livre de lecturé courante par les Frères du Sacré-Cour, cours supérieur, 2e éd., Montréal, Les Frères du Sacré-Cœur, 1912, 376 p., ill. 
reproduisent les principaux dogmes du christianisme, rappelant ainsi aux élèves ceux qu'ils apprenaient dans leur catéchisme. Voici le nombre de gravures illustrant des scènes religieuses: syllabaire, 13 $(76 \%)$; cours élémentaire, $28(59 \%)$; cours moyen, $43(74 \%)$; cours supérieur, $25(37 \%)$. Comme on le constate. elles sont en nette régression dans le cours supérieur où l'accent patriotique l'emporte.

Ces dessins sont l'œuvre des FF. Joseph, Loyola, (Stanislas) et MM. A. Morissette et Edmond Massicotte. Les scènes décrites s'inspirent parfois de l'histoire religieuse canadienne. On retrouve, sur la couverture, le petit Saint Jean-Baptiste avec sa croix et son mouton. Il y a aussi NN.SS. Laval et Briand, les PP. Brébeuf et Marquette. Le sanctuaire de Ste-Anne-de-Beaupré y est représenté ainsi que les célèbres zouaves canadiens. Les autres illustrations mettent en relief l'histoire du christianisme en France avec les Bossuet, Saint Vincentde-Paul, Sainte Jeanne d'Arc et le sanctuaire de Lourdes.

La sélection des écrivains révèle assez justement l'idéologie des responsables des manuels concernés et du milieu canadien-français. D'origine française ou canadienne-française, les auteurs cités incarnent un christianisme conservateur. De plus, le but poursuivi par les auteurs des manuels reflète l'atmosphère religieuse de l'époque. Ils veulent «fournir aux élèves qui quittent les tableaux, des sujets de lecture, religieux, attrayants, moraux et instructifs» 25 . Dans la préface du cours moyen, ils déclarent aux maîtres qu'une «large part a été faite à la morale, à la tempérance surtout. aux leçons de choses, à la poésie et à l'agriculture » ${ }^{26}$.

Ainsi les sujets religieux étaient abordés de manière doctrinale ou morale. Les premiers se rapportaient à Dicu et aux vérités enseignées par l'Église. Les seconds proposaient une conduite exemplaire aux chrétiens vivant dans la société. S'adressant surtout à l'adulte en puissance dans l'enfant, les sujets étaient traités d'une manière théologique, canonique, sans lien avec la Bible. Seules quelques citations de l'Ancien Testament et de l'Évangile viennent rappeler les dimen-

25 Livre de lecture courante par les Frères du Sacré-Caur. cours élémentaire, $3^{*}$ éd., Montréal, Les Frères du Sacré-Cœur, 1910, p. 3. À l'avenir. on ne retiendra que la partie essentielle du titre de ces manuels: Livre de lecture counante, cours élémentaire....

26 Livre de lecture courante, cours moyen. (2e éd.), Montréal, Les Frères du Sacré-Cœur, 1897, p. 6. 
sions de l'histoire du salut, c'est-à-dire l'enracinement historique de la foi chrétienne.

Les éléments de l'enseignement chrétien que renferment les livres analysés sont faciles à déterminer. Tout d'abord, Dieu y est présenté comme l'Être suprême : «Dieu est grand, Dieu est bon, Dieu est Saint ${ }^{27}$. Par sa Providence, il dirige l'homme et le monde car «Il voit tout, il sait tout, et il peut faire tout ce qu'il veut» ${ }^{28}$. À la vue de ses bienfaits « un cri de reconnaissance doit s'échapper de nos cœurs pour bénir et remercier la Providence qui veille sur nous avec tant de sollicitude $\gg 29$. En outre, Dieu se sert des anges pour guider les hommes dans la bonne voie. L'ange gardien mérite une bonne prière pour la protection qu'il accorde à l'enfant :

Veillez sur moi quand je m'éveille

Bon ange, puisque Dieu l'a dit :

Et chaque nuit, quand je sommeille,

Penchez-vous sur mon petit lit 30 .

La dévotion à la Vierge est fortement recommandée : pèlerinages, images, chapelets et scapulaires. "Portez-les (chapelets et scapulaires) donc toujours sur vous avec confiance et amour si vous voulez que Marie se montre véritablement votre Mère ${ }^{31}$. Quant aux nombreux saints toujours grandis par leurs gestes miraculeux, ils retiennent facilement l'attention des adolescents.

Les martyrs sont les premiers saints de cette Église catholique dont nous avons déjà parlé. Nous devons honorcr leur courage de notre reconnaissance et de notre admiration. C"est peut-être parce quïls ont souffert avec constance qu'aujourd'hui nous avons le bonheur d'être chrétiens. Plutôt que d'offenser Dieu par le péché mortel, ils ont enduré les tourments les plus affreux et la mort la plus cruelle ${ }^{32}$.

27 Livre de lecture courante, cours élémentaire, 3e éd., Montréal, Les Frères du Sacré-Cœur, 1910, p. 6.

28 Ibid.

29 Livre de lecture courante, cours moyen, (2* éd.), Montréal, Les Frères du Sacré-Cœur, 1897, pp. 73-74.

30 Livre de lecture courante, cours élémentaire, $3^{\text {trd }}$ é, Montréal, Les Frères du Sacré-Cœur, 1910, p. 26. Texte à mémoriser.

31 Liv're de lecture courante, cours moyen, (2e éd.), Montréal, Les Frères du Sacré-Cœur, 1897, pp. 42-43.

32 Ibid., p. 138. 
De son côté, l'Église a la responsabilité de conduire le fidèle au salut malgré toutes les embûches :

Vous savez que le premier pape de l'Église catholique fut saint Pierre, d'abord pêcheur de profession. C'est pour cela que l'Église est représentée par une barque que les impies s'efforcent de faire sombrer, mais qui voguera victorieusement sur les flots mugissant jusquı'à la fin du monde 33 .

Parmi les autres moyens mis à la disposition du fidèle pour suivre le Seigneur Jésus, la prière occupe une place de première importance :

Un bon chrétien ne manque pas de prier le matin. le soir et souvent dans la journée. Il le fait encore d'une manière plus pressante lorsque quelque danger le menace ou que le démon cherche à porter la mort dans son âme.

Après que vous avez prié, ne sentez-vous pas votre cœur plus heureux? Vous êtes des voyageurs ou des exilés cherchant la patrie; il faut donc souvent lever les yeux pour reconnaitre votre route. Or, la patrie, c'est le ciel, où vous parviendrez surtout par la prière ${ }^{34}$.

En plus de la partie doctrinale du catholicisme, la vie morale du chrétien constitue un aspect important de l'enseignement religieux. Dans les manuels étudiés, les thèmes moraux se présentent sous deux formes différentes, comme l'a aussi constaté Pierre Zind dans les manuels de lecture courante publiés en France au XIX siècle ${ }^{35}$. Dans le premier cas, le texte est imprégné de la morale catholique. Dieu y est nommé et l'argumentation repose sur la conception chrétienne de la vie. Dans certains cas, lorsque l'accent religieux du morceau n'est pas assez prononcé, on se permet d'y ajouter une réflexion à la fin. Ces éléments se retrouvent dans un texte sur la santé où l'on rappelle, en premier lieu, les merveilles du corps, le besoin d'une saine alimentation et l'importance d'une vie réglée. Mais la conclusion moralisante ne tarde pas à mettre en relief la vie chrétienne comme gage d'une santé florissante :

Mais le plus sûr moyen de jouir d'une bonne santé. c'est de vivre bien chrétiennement. Presque toutes les maladies ont

33 Ibid., p. 109.

34 Livre de lecture courante, cours moven, (2e éd.), 1897, Montréal, Les Frères du Sacré-Cœur, p. 145.

35 Pierre ZIND, L'enseignement religieux dans l'instruction primaire publique de 1850 à 1873, Lyon, Centre d'histoire du catholicisme, 1971, 314 p., pp. 116-119. 
leur origine dans quelque vice proscrit par l'Évangile. Il y a des maladies qui viennent de la paresse, d'autres de la colère, d'autres de la gourmandise, etc.

Vivre selon la loi de Dieu, c'est du même coup se précautionner le plus possible contre les infirmités et les maladies ${ }^{36}$.

Selon l'autre forme, la conduite décrite fait appel à la morale dite naturelle; il n'y a aucune référence à la religion catholique. Le comportement social exprimé s'appuie sur une conception laïque de la société. Le texte de L. de Ratisbonne racontant une distribution de prix est conçu dans cet esprit. La direction d'une école de village veut décerner un prix de santé au petit Thomas mais celui-ci le refuse prétextant qu'il n'a fait aucun effort pour cela. Fort de cet exemple, l'auteur fait ressortir la primauté de l'effort sur la facilité :

Dans la vie, comme à l'école, c'est la peine apportée, c'est l'obstacle vaincu, c'est l'effort fait qui mérite la couronne. Pour être vainqueur, il faut avoir lutté ; pas de triomphe vrai, si le hasard seul le donne; car où il n'y a eu ni travail, ni peine, il ne saurait $y$ avoir de gloire ${ }^{37}$.

Mais c'est en dressant le portrait de la vie morale du chrétien tel qu'il ressort des textes de ces manuels que nous comprenons mieux l'enseignement donné aux jeunes. L'enfant ne doit pas négliger son instruction :

De nos jours, mon petit ami, il n'est plus permis d'être ignorant; il faut une certaine somme d'instruction pour faire honorablement son chemin dans la vie. Sachez donc profiter de vos années d'école pour devenir un homme instruit, et surtout un bon chrétien qui serve Dieu, l'Église, la patrie, qui soit l'honneur de sa famille et le modèle de ses concitoyens ${ }^{38}$.

Il doit aussi obéir à ses parents, pratiquer la charité envers ses compagnons, protéger les animaux, être franc, aimer le travail ${ }^{39}$. Dès maintenant et lorsqu'il sera adulte, il surveillera ses lectures parce qu'elles exercent "sur la plupart des hommes une influence prédominante », et suivra les conseils de l'Église en cette matière :

N'achetons donc et ne lisons que de hons livres, que des journaux franchement catholiques; et, non contents de nourrir

36 Livre de lecture courante, cours moyen, (2e éd.), Montréal, Les Frères du Sacré-Cœur, 1897, p. 103.

37 Ibid., p. 105.

38 Ibid., p. 8.

39 Ibid., pp. 51-53, 26-27, 86-88, 118. 
notre âme de bons principes, faisons-nous les propagateurs dévoués de la presse honnête et religieuse ${ }^{40}$.

En outre, le futur citoyen devra éviter l'intempérance : l'alcool est "un véritable fléau pour l'individu qu'il abrutit et qu'il tue, pour la famille qu'il désorganise et qu'il réduit souvent à la plus affreuse misère physique et morale, pour la race qu'il appauvrit et la société qu'il ruine ${ }^{4 l}$.

Telle apparaît l'image de l'adolescent modèle. Il est à signaler la différence marquée entre les manuels publiés en 1897 et 1910 quant aux textes moraux. Dans le cours élémentaire et moyen, ils représentent $45.3 \%$ et $43.8 \%$ de l'ensemble des thèmes abordés, tandis que dans le cours supérieur, ils ne constituent que $16.9 \%$. Ce changement est-il dû à une mutation de la société canadiennefrançaise ou simplement à un souci d'adaptation pédagogique des auteurs ? Comme nous le remarquerons, à compter de 1910, les textes à résonances patriotiques recevront une plus large diffusion au détriment des textes moralisants.

\section{III - LE NATIONALISME DANS LES MANUELS}

L'étude du nationalisme dans les manuels de lecture courante nous permet de connaître l'enseignement patriotique donné aux élèves au début du $X^{e}$ siècle. Sans être des manuels d'histoire, ils renferment plusieurs textes d'historiens et d'écrivains canadiens. Notre approche consistera à dégager les grands traits que revêt le nationalisme canadien-français. De nouveau la religion sera mise en évidence car elle fait partie intégrante du nationalisme canadien-français.

Le nombre d'illustrations qui rejoignent les thèmes sociaux, en grande partie nationalistes, n'est pas le même dans les différents manuels étudiés. Dans celui de 1910, se ressentant sans doute de l'atmosphère nationaliste entretenue par Bourassa et la Ligue nationaliste ${ }^{42}$, l'importance accordée à l'illustration des thèmes patrio-

40 Livre de lecture courante, cours supérieur, $2^{\mathrm{e}}$ éd., Montréal, Les Frères du Sacré-Cœur, 1912, pp. 300-302. Texte extrait de la Semaine religieuse d'Arras.

41 Ibid., p. 362. Texte du Dr. Rouxel.

42 Voir Joseph Levitt, Henri Bourassa and the Golden Calf. The Social program of the Nationalists of Quebec, 1900-1914, Ottawa, Éd. de l'Université d'Ottawa, 1969. Coll. Cahiers d'Histoire, no 3. 
tiques est révélatrice. Il en va de même pour les nombreuses lectures portant sur la société : cours élémentaire, 18 (18\%); cours moyen, 19 (16\%) ; cours supérieur, 78 (53\%). Cette progression est encore plus frappante si l'on considère l'origine ethnique des auteurs cités. Cette répartition donne les résultats suivants : cours élémentaire (1897), 21 Français et 1 Canadien ; cours moyen (1897), 39 Français et 20 Canadiens ; cours supérieur (1910), 48 Français et 47 Canadiens. Il faut noter la progression constante d'auteurs canadiensfrançais. La date de publication des manuels n'est pas étrangère à cette accentuation du nationalisme, à cette «canadianisation ». L'analyse idéologique de ces auteurs, tant clercs que laïcs, serait intéressante mais elle dépasse le cadre de cette recherche.

Les sujets sociaux se partagent en deux grandes catégories: ceux relevant du nationalisme et ceux ayant trait aux connaissances usuelles. Dans ce chapitre, seuls les thèmes patriotiques retiendront notre attention car, mieux que les connaissances usuelles, ils touchent la survie du peuple canadien-français. Quant à l'insistance donnée aux sciences, elle dénote un souci de se tourner vers une société de plus en plus industrialisée et technique.

\section{Le rôle de l'Église dans l'histoire du Canada}

L'Église apparaît, grâce à sa pérennité, comme la sauvegarde des valeurs les plus fondamentales des Canadiens français :

Hâtons-nous de le proclamer, c'est à l'Église catholique que nous devons notre conservation comme race distincte et le merveilleux épanouissement de nos forces, sur la moitié de ce continent.

Debout sur le berceau comme sur la tombe des nations, l'Église préside aux destinées des peuples. Du haut de son immortalité, elle les voit naître et mourir au pied de ce rocher sur lequel elle a été fondée par une main divine. Aussi, nous n'aurions rien à craindre pour notre avenir tant que la barque de l'Église portera le sort de notre nationalité ${ }^{43}$.

\section{L'action du clergé est à cet égard déterminant :}

Quoique exempt par état de se mêler activement de politique, notre clergé nous a rendu, sous ce rapport, d'incontestables services dans le cours de nos grandes luttes. On lui a quelque-

43 Livre de lecture courante, cours supérieur, $2^{\mathrm{e}}$ éd., Montréal, Les Frères du Sacré-Cœur, 1912, pp. 323-324. Texte de L.-A. Prud’homme. 
fois reproché d'être trop timide, mais combien de mouvements populaires irréfléchis n'a-t-il pas empêchés ou restreints? Combien d'œuvres publiques et nationales n'a-t-il pas favorisées? Combien d'utiles conseils et d'encouragements n'a-t-il pas donnés à nos hommes publics dans les temps difficiles ? 44

Les Canadiens français ont une mission providentielle à accomplir sur le continent américain : maintenir lạ foi et la langue française, gages de leur vitalité :

Nous avons conquis toute la province de Québec, envahi la partie orientale d'Ontario, refoulant devant nous les autres nationalités et nous nous sommes frayé un chemin jusque dans les prairies de l'Ouest, où des essaims nombreux se sont enracinés dans le sol, avec la ténacité traditionnelle de notre race... Tout peuple a reçu de Dieu une mission spéciale et lorsque ce peuple lui demeure fidèle et suit avec droiture la voie qu'il lui indique, il le bénit avec amour et le couvre de son bras protecteur ${ }^{45}$.

\section{Les grandes étapes de l'histoire du Canada}

Les événcments marquants de l'histoire d'un peuple lui reviennent constamment à la mémoire. Le peuple canadien-français n'échappe pas à ce phénomène naturel. Dans son passé, la NouvelleFrance lui apparaît comme chargée de signification, de symbolisme, car elle renferme les caractéristiques les plus essentielles de son existence, telles la foi, la langue et la patrie.

La période de colonisation en Nouvelle-France est considérée comme une œuvre inspirée par la Providence. Les pionniers vivaient une ère de ferveur malgré les embûches de toutes sortes créées par les Indiens :

Le même esprit animait les simples laïques et les religieux, les femmes faibles et délicates, aussi bien que les soldats et les hardis explorateurs qui s'aventuraient au milieu des tribus sauvages. Que de nobles natures se sont développées parmi les Français du Canada, dans la lutte entre la civilisation chrétienne et le naturalisme sauvage des aborigènes ${ }^{46}$.

44 Livre de lecture courante, cours moyen, (2e éd.), Montréal, Les Frères du Sacré-Cœur, 1897, p. 196. Texte d'Étienne Parent.

45 Livre de lecture courante, cours supérieur, $2^{\mathrm{e}}$ éd., Montréal, Les Frères du Sacré-Cœur, 1912, p. 323. Texte de L.-A. Prud'homme.

46 Livre de lecture courante, cours moyen, (2e éd.), Montréal, Les Frères du Sacré-Cœur, 1897, p. 199. Texte de J.-B.-A. FerLand. 
Descendants de valeureux pionniers, les Canadiens français peuvent être fiers, selon $\mathrm{M}^{\mathrm{gr}}$ Laflèche, de ces premières familles de la colonie, "choisies parmi ce qu'il y avait de mieux dans la mèrepatrie, sous le rapport moral et religieux ${ }^{47}$.

Certains héros retiennent davantage l'attention : Cartier, Champlain, Maisonneuve, "[dont la] mission providentielle est un fait tellement acquis à l'histoire que vouloir la révoquer en doute serait de la témérité ${ }^{48}$, Dollard enfin et ses compagnons, dont le courage est propre à stimuler les jeunes : avant de partir pour la bataille, «ils firent leur testament, se confessèrent et communièrent ensemble, et, en présence des saints autels, promirent de ne jamais demander quartier et de verser leur sang pour la religion et le salut de la patrie ${ }^{49}$.

La bataille des plaines d'Abraham vient clore l'épopée du Régime français. Le rôle de Montcalm, même dans la défaite, apparaît grandiose. «Malgré la violence du mal il resta à cheval, et, soutenu par deux grenadiers, il rentra dans la ville consternée, où il mourut le lendemain matin, avec tous les sentiments d'un véritable héros chrétien » 50 Cette défaite amenait un tournant dans l'histoire du Canada. Qu'allaient devenir les colons français établis sur les bords du Saint-Laurent?

Ces généreux enfants d'une race superbe Seraient-ils absorbés par le peuple vainqueur?

- On le disait. Mais Dieu, qui prend soin du brin dherbe Laissait luire sur eux un rayon de son cœur ${ }^{51}$.

De la Conquête à la Confédération, peu d'événements inspirent les écrivains cités dans les manuels. Le seul personnage politique qui retient l'attention est Hippolyte LaFontaine. L'avènement de la Confédération canadienne donnant à chaque province une plus grande autonomie enchante A.-B. Routhier. Le livre de lecture cou-

47 Livre de lecture courante, cours supériéur, 2" éd., Montréal, Les Frères du Sacré-Cœur, 1912, p. 293.

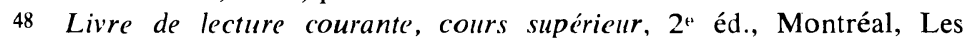
Frères du Sacré-Couur, 1912, p. 306. Texte de N.-E. Dionne.

49 Ihid., p. 118.

50 Ihid., p. 292. Texte de C.-H. Laverdière.

51 Ibid., p. 359. Texte de A.-B. Routhier. 
rante du cours supérieur reprend les vers que lui inspire la date de 1867 :

Ah ! c'est que, confiante en sa force admirable,

La nation affirme aujourd hui ses succès :

C'est qu'elle voit s'ouvrir, en ce jour mémorable,

Dans les murs de Québec un parlement français! 52

Les Louaves canadiens-français émerveillaient les jeunes qui les voyaient défiler lors des cérémonies patriotiques et religieuses : "Les hommes courageux qui ont offert et exposé leur vie à la défense du trône de Saint Pierre, méritent doccuper un poste honorable dans le sanctuaire $\gg 53$. En France, ces hommes avaient attiré l'attention, et lors de leur passage à Lyon en 1868, M. Victor de Laprade, membre de l'Académie française, leur avait adressé les vers suivants, repris dans le manuel:

Allez votre chemin, Français du Nouveau-Monde!

Race de nos aïeux tout à coup ranimés,

Allez, laissant chez nous une trace féconde

Offrir un noble sang au Dieu que vous aimez...

Libres soldats, nourris près d'une république

Fils d'une terre où l'homme a toute sa fierté,

Vous témoignez,au nom de la jeune Amérique

À la fois pour le Christ et pour la liberté 54 .

\section{La patrie, une des sources du nationalisme canadien-français}

Tous ces devanciers ont contribué à édifier la patrie. En elle se rejoignent le passé, le présent et l'avenir. Elle est le lieu de convergence par excellence du nationalisme. Les exemples de cette prise de conscience patriotique ne manquent pas surtout dans le manuel de lecture édité en 1910: "Nous devons aimer notre patrie : c'est là que nous sommes nés, que nous avons grandi, que nous avons aimé ; c'est là que reposent nos aïeux et c'est là que Dieu nous a placés $» 55$.

À cette époque, le patriotisme canadien-français est toujours envisagé dans une perspective canadienne. Il ne se restreint pas au

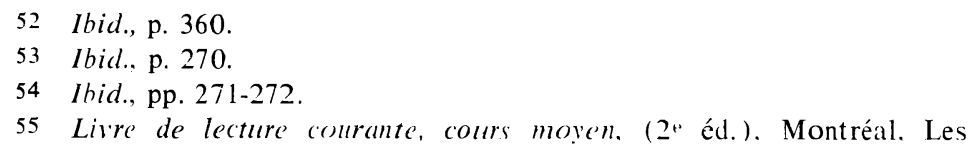
Frères du Sacré-Cœur. 1897, p. 92. 
Québec même si le territoire de cette province constitue le pivot de toute son affectivité nationale. La conquête du sol, la colonisation a été l'instrument principal d'implantation des Canadiens français dans les différentes régions du Québec. Le livre de lecture reprend un poème d'Adolphe Poisson qui chante la conquête des Cantons de l'Est :

Un jour, en contemplant la plaine déboisée,

L'étranger refoulé frissonna de stupeur.

Ô champs qu'on a peuplés d'une autre race altière

Cantons de l'Est dotés, hélas ! d'étranges noms,

Vous qui deviez servir contre nous de harrière,

Vous nous apparteniez et nous vous reprenons ${ }^{56}$.

De son côté, le métier d'agriculteur est le plus favorable à une vie chrétienne exemplaire :

Puisque la culture de la terre est imposée par Dieu, il faut que ce soit quelque chose de bien grand et de bien honorable. Voilà pourquoi nous devons estimer et chérir la profession d'agriculteur. C'est une profession utile et honorable entre toutes, et je ne crois pas quil existe en ce monde. si ce n'est le sacerdoce, un emploi ou plus ancien. ou plus nécessaire et plus noble 57 .

Les auteurs des manuels étudiés insistent sur les connaissances nécessaires en agriculture. On comprend mieux dès lors cette phrase d'une des préfaces: "La part considérable que nous avons faite à l'agriculture sera, nous n'en saurions douter, bien appréciée de tous ceux qui comprennent que l'avenir et la prospérité de notre pays reposent sur le développement et la diffusion de la science agricole ${ }^{58}$. En outre, l'agriculture est la gardienne d'un mode de vie. Loin des villes, les coutumes se sont gardées intactes. Par exemple, le réveillon de Noël a gardé son cachet d'antan que les citadins ne doivent pas mépriser :

Sybarites de salons, enfants pâles de villes, qui vous croyez heureux de vivre douillettement dans vos logis chauffés par

56 Lirre de lecture courante, cours supérieur. 2" éd., Montréal, Les Frères du Sacré-Cœur, 1912, pp. 289-290.

57 Liver de lecture courante, cours supérieur, 2" éd., Montréal, Les Frères du Sacré-Cœur, 1912. p. 130. Texte anonyme.

58 Livre de lecture courante, cours moye'n, (2" éd.), Montréal, Les Frères du Sacré-Cœur, 1897, p. 6. 
d'invisibles calorifères, par la vapeur et le gaz, par le feu sombre du coke et du charbon, ou bien encore par quelques boîtes à feu; qui grignotez du bout des lèvres quelques délicates friandises, ne riez pas des bons réveillons d'habitant. devant le gros poêle, où se consument de bonnes grosses bûches de bois franc, au milieu d'un grahat de charbonnailles ardentes, cependant que l'on entend le boudin. la saucisse et loịe grasse grésiller dans les fourneaux . . . 59

La patrie se construit à partir d'une langue. Intimement liée à la vie d'un peuple, la langue est à cette époque le meilleur rempart de la patrie et de la religion. Le poète William Chapman a mis en évidence le caractère nationaliste de la langue française pour les Canadiens français. Elle a été liinstrument d'évangélisation, et malgré les affronts qu'elle a subis, elle n’en demeure pas moins le signe de sa survivance :

Nous avons conservé liidiome légué

Par ces héros quittant pour nos bois leurs falaises

Et. bien que par moments on le crût subjugué.

Il est encore vainqueur sous les couleurs anglaises 60 .

Le nationalisme a besoin de symboles pour s'extérioriser. À ce titre, le drapeau concrétise les aspirations patriotiques d'un peuple. Pour les Canadiens français, le drapeau «Carillon Sacré-Cœur» réunissait tous les éléments de leur histoire : le rappel de la victoire de Montcalm, la feuille d'érable, la couronne et le cœur :

Sur mon drapeau, comme dernier symbole

Plus beau qu'un lis, plus brillant que l'or pur,

Sur mon drapeau je veux une auréole

S'irridiant en rubis dans l'azur.

Du Golgotha Jésus brise la pierre,

Dompte le monde et son rire moqueur.

Et conquérant le palais, la chaumière.

Roi légitime, il s'avance en vainqueur.

Sur mon drapeau. qui marche à ta lumière,

Ô Christ, ô Roi, je veux ton divin Cœur ${ }^{61}$.

59 Livre de lecture courante, cours supérieur, 2" éd., Montréal, Les Frères du Sacré-Cœur, 1912, p. 321. Extrait de "Restons chez nous», de D. Potvin.

60 Ibid., p. 332.

61 Livre de lecture courante, cours moyen. (2e éd.), Montréal. Les Frères du Sacré-Cœur, 1897, pp. 202-203. Texte à mémoriser. 
Le symbole atteint sa pleine signification lors d'une manifestation collective. À l'occasion de la Saint-Jean-Baptiste, fête patronale des Canadiens français, le peuple revit alors tout son passé. C'est l'occasion de rappeler son rôle en Amérique :

Fidèle aux traditions du passé, fier de ses libertés actuelles, confiant en sa force vitale, le Canadien aspire à devenir un peuple indépendant, capable par lui-même de se défendre des attaques de ses puissants voisins. Il aspire à devenir un peuple digne de jouer en Amérique le rôle de bienfaisance sociale et humanitaire que la France chrétienne n'a cessé d'exercer en Europe jusque dans ces dernières années. Son idéal est encore plus élevé. Il aspire à conserver, à développer dans son cœur, à répandre autour de lui, les trois flammes qui embrasent son âme : l'amour de la patrie, l'amour de l'Église et l'amour du Sacré-Cœur 62.

En conclusion, on peut se demander si les thèmes religieux et nationalistes proposés à la jeunesse étaient bien adaptés à la mentalité des Canadiens français. S'agissait-il d'une production étrangère dont l'enracinement en terre canadienne n'était que minime ? Il faut dire que ces volumes étaient en grande partie d'inspiration française. Le syllabaire et le cours élémentaire sont des livres importés au Canada car ils correspondent à une édition publiée en France ${ }^{63}$. Seule l'illustration a été augmentée et le texte de forme dialoguée a cédé la place au récit. Quant aux manuels du cours moyen et supérieur, ils sont aussi dans une large mesure le calque d'une édition française ${ }^{64}$. Mais la forte dose d'éléments nationalistes contenus dans le manuel du cours supérieur révèle une adaptation à l'évolution du milieu canadien-français.

L'attitude des religieux enseignants qui utilisaient ces manuels est difficile à déterminer. Les notes d'appréciation sont rares et il faudrait procéder par interviews pour suppléer à cette lacune. Il en

62 Ibid., p. 203. Texte identifié par les initiales G.L.

63 André COINDRE, Livre de lecture courante, cours élémentaire, Toulouse. Imp. et Librairie Édouard Privat, s.d., Coll. : Livres primaires.

64 Celle-ci est parue en un seul volume: F.N., Livre de lecture courante, premières notions sur les sciences, cours moyen et supérieur, Toulouse, Édouard Privat et Paradis (près Le Puy), Procure générale, 1894. Coll.: Classiques F.N. 
est de même pour les élèves dont les réactions auraient été intéressantes à connaître pour évaluer l'influence des sujets abordés. En ce qui concerne les professeurs, existait-il une différence d'appréciation entre les Frères canadiens et français à ce sujet ? Pour ces derniers encore nombreux au Canada et détenant tous les postes clés de l'Institut vers 1900 - ils devaient réagir favorablement face à ces manuels dans lesquels ils avaient peut-être étudié. Le patriotisme canadien-français ne les rapprochait-il pas de leur pays d'origine? Arrivés de plein gré ou obligés de s'exiler lors des persécutions religieuses de 1900 à 1905, ils devaient vibrer en relisant ces textes tout empreints de l'histoire de la France, expliquer avec beaucoup de verve les hauts faits de la mère patrie envers sa colonie. De plus, cette nouvelle patrie présentait plusieurs traits familiers à la France d'antan : rôle dominant de l'Église en éducation, présence de l'ultramontanisme et caractère français de la population.

Ces dernières questions suscitent d'autres recherches qu'il faudrait poursuivre pour une meilleure compréhension du milieu québécois du début du $X^{\prime}$ siècle. Une étude approfondie de l'idéologie des manuels du niveau primaire ou secondaire publiés en France et au Canada se révélerait très intéressante. En outre, jusqu'à quel point la venue des communautés religieuses françaises a-t-elle contribué à maintenir et à renforcer les liens entre Canadiens français et Français? Il serait aussi révélateur de mesurer les différences et les ressemblances au plan pédagogique et idéologique entre les divers manuels publiés par les congrégations religieuses au Canada français. Ainsi on pourrait déterminer les apports pédagogiques de chaque Institut et surtout préciser la vitesse de la «canadianisation» de ces communautés. L'aspect économique du livre scolaire avec, entre autres, les conflits qu'il a suscités entre le gouvernement provincial et les communautés religieuses reste à écrire. Enfin, une comparaison entre les manuels publiés au Québec et en Ontario serait révélatrice de la conception de l'éducation et des idéaux sociaux chez les francophones et les anglophones du Canada.

Jacques PARAdIs, Polyvalente La Magdeleine, Laprairie. 